\title{
Enhanced Routing Protocol for VANET
}

\author{
Ramneet Kaur, Dr.Navdeep Kaur,Dr.Satwinder singh
}

\begin{abstract}
A class of networks called VANET(vehicular adhoc network), the extension of MANETs(mobile adhoc network) are based on the principle of the formation of wireless network for exchange of data and the creation of network is spontaneous in nature. The mobility constraints, behavior of driver, high speed, limited coverage of wifi, hard delay constraints leads to unique characteristics in VANETS. So the MANET routing protocols are not suitable for VANET. Optimization of routing protocols becomes necessary to make it suitable for VANET. In this paper, various optimized routing protocols are analyzed and their optimization techniques are discussed. Parameters such as end to end delay and energy spent are taken into consideration in order to show the improvement from the routing protocols that are standard protocols. Then a method has been proposed to optimize the fine tuned OLSR (optimized link state routing)protocol with the use of advanced genetic algorithm to further improve the results and to make the protocol more efficient.
\end{abstract}

Index Terms:Optimized link state routing (OlSR), particle swarm optimization (PSO), Quality of service (QoS), Vehicular Adhoc Network (VANET).

\section{INTRODUCTION}

Vehicular Adhoc Network(VANET) is a network that supports the communication of one vehicle with other vehicles that are near to it or roadside units that are installed in centralized locations. There are two types of communication: vehicle to vehicle and vehicle to infrastructure. In vehicle to vehicle communication, short range wireless technologies such as wifi and wave are used to exchange data. In Fig 1. Vehicles are equipped with electronic devices that allow them to receive or relay messages. In Vehicle to Vehicle communication vehicles are equipped with on board units that are used to transmit a message. Vehicles move in organized manner in accordance to traffic rules, traffic signals, limits and speed signs. Various routing protocols are used by vehicles to exchange messages .Topology related information is exchanged by routing protocols in order to find out an efficient path between the vehicles. Routing protocols are divided into following groups:(1) position based routing protocol uses nodes location information by using global positioning system (GPS), instead of links information to routing[14][16]. In position based routing Protocols, the packet source node has position information of its own and the packet destination node and position information of neighbors

\section{Revised Manuscript Received on June 15, 2019.}

Ramneet kaur, Computer Science,Shri GuruGranth Sahib World University, fatehgarh Sahib, India.

Dr.Navdeep Kaur, Computer Science,Shri Gur Granth Sahib World University, fatehgarh Sahib, India.

Dr.Satwinder Singh, Computer Science,central University of Punjab,Bathinda,India .

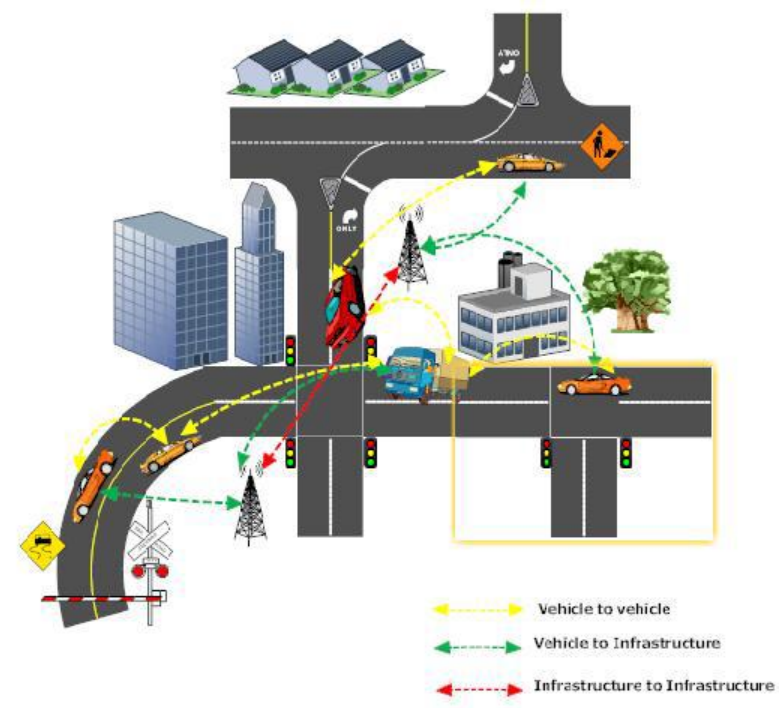

FIG 1. VEHICULAR ADHOC NETWORK

. For example (2) Topology based routing protocol the topology information is stored in tables and updated from time to time.(3)Cluster based routing protocol divides the area into grids and cluster head is selected that holds the responsible for inter and intra cluster coordination. (4) Broadcast routing protocol such as BROADCOMM sends packets to every node in the network. (5)Geocastrouting protocol such delivers packets to vehicles located in a specific static geographic region.

\section{CHARACTERSTICS OF VANET}

The behavior of driver, mobility constraints paves a way to extraordinary traits. The main traits are as follows:

Rapid changing topology and high mobility: Movement of vehicles is very fast especially on highways.Connections are born and broken immediately because vehicles stay in communication range for few seconds.In the low density, chances of disconnection of vehicles is high[17].

Geographic position available: Vehicles can be provided with electronic maps with the help of accurate positioning systems. For instance, GPS chips in cars helps to generate position information for purpose of routing.

Mobility modeling and prediction: Vehicular nodes are usually restricted by highways that are estabalished before hand, vehicles future position can be forecasted by giving the speed .

Hard delay constraints: Collision alert or crash Sensing are the vanet applications in which the environement need not to have increased information rates but is restricted by difficult delay.

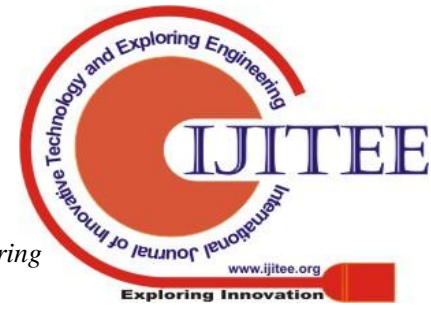


No power constraint:Power constraint can be ignored since hubs are moving vehicles not stable devices and batteries are always recharging .

\section{RELATED WORK}

\section{A. Routing Algorithms in VANET}

The most important issue in VANET is the design of efficient routing protocol.

In ,Yun-wei lin et al.[5] has surveyed various routing protocols and introduced unicast protocol[11], multicast protocol geocast protocol,mobicast protocol and broadcast protocol[5]. Carry and forward technique has been considered as the key for designing all the routing protocols. The unicast routing protocol is divided into:

min-delay unicast routing protocol constructs a path with minimum delay .Transmission delay is the major issue so the shortest path is adopted .In case of low density area when there is no neighboring vehicle to forward the packet ,multihop forwarding cannot be used .carry and forward technique can be utilized.

Delay bounded routing protocol provides a scheme that maintains low level channel utilization while satisfying user defined delay requirements.

They have discussed temporary network fragmentation problem that is caused by rapidly changing topology and broadcast storm problem that affects the rate of message delivery in VANET.They have also discussed challenges and future perspectives of routing protocols for VANETs. A comparison of routing algorithms has been done by considering various parameters[5].

\section{B. Optimized Routing Algorithms}

The routing protocols in VANET is plagued by a major problem of end to end delay and data loss due to loss of connection among highly mobile vehicles. By finding the shortest path a packet can be sent quickly to its destination before the vehicle gets disconnected.

Radhika kochar and hardwarilalmandoria [3] have considered biological ants for their study. The principle is to use ants like packets and to gather routing information from their operation the main aim is to find out the shortest path from source to destination they have used vehicle's movement pattern, vehicle velocity, vehicle density, direction and time conditions for the analysis .NS2 is used to simulate AODV routing protocol. The results produced shows reduction in end to end daley.They have concluded that ant based approach is powerful means to solve routing problems in VANET.

Himani rana et al.[8]have proposed swarm based hybrid routing algorithm. It is basically multipath routing algorithm. Vehicles are divided into zones. Reactive routing is applied between zones whereas proactive routing is applied within zone. The algorithm produced better delivery ratio and reduced end to end delay and significant improvement in packet delivery ratio . The algorithm is more suitable for dense scenario. They have used the vehicle position and speed to make predictions on the mobility of the vehicles[10].NS2 is used to simulate mobility aware zone ant colony optimization routing Hence it is concluded that hybrid ACO algorithm is scalable and achieves good connectivity of network.
Uday mane et al. [4] in their paper has emphasized on improvement of the QoS parameters in VANETs. The wireless routing protocols such as AODV, AOMDV and OLSR is analyzed using ns2. Ant colony optimization has been applied to AODV.A considerable improvement in delay has been shown.

Allison et al. [12 ] to make stable tree based multicast routing have applied principles based on ant colony and mobility prediction. A path with greater lifetime and smaller hop counts is used by multicast tree that is built by MAODV . It has been observed that MAVAODV gives better performance than MAODV. The MAODV satisfies the objective of maintaining the multicast tree based on higher level of connectivity in limited computational time.

Srinjoy Ganguly and Swagatam Das[1] proposed novel pheromone deposition, local search \& mutation strategies in order to solve vehicle routing problem efficiently .A novel sequential constructive local search operator has been proposed that leads to fitter solutions and faster. The Vehicle Routing Problem (VRP) is one of the most important problems in the area of Operations Research. To solve the Vehicle Routing Problem a novel Ant Colony Optimization algorithm is presented in this paper. By virtue of this algorithm the authors have proposed novel pheromone deposition, local search \& mutation approaches to solve the VRP and to provide rapid convergence. The results provided by ACO_PLM are better as compared to other heuristics, which is clear from the experimental results.

GUO Zhong-hua, SHI Hao-shan[6] puts forward the optimization of DSR (dynamic source routing) protocol with the use of constrained dynamic query localization approach called LDSR protocol in order to solve the routing overhead problem of the Ad Hoc network DSR protocol formed as a result from flooding. Dynamic choice mechanism is used by LDSR protocol in order to constraint query to a small region and full flooding localization which is based on original DSR protocol and constrained by two factors: (1) the small-world theory determines the maximum number of hops of query localization flooding; (2) mobile critical transmitting range determines the effective routing time for connectivity in Ad Hoc network and the maximum mobile speed of node. simulation results have shown that LDSR protocol can produce better average end-to-end delay ,but packet delivery fraction decreased as compared to DSR protocol

Anusha bandi and Chandrashekhar[7]have proposed an optimization strategy in order to fine-tune few metrics like delay, packet delivery ratio by enhancing the OLSR protocol with the help of particle swarm optimization. The few metrics has been taken into account in order to compare the values of fitness,packet delivery ratio and throughput.In the end particle swarm optimization algorithm has been compared with genetic algorithm by taking into account QoS parameters.

\section{Security}

The most challenging areas in todays research is to securely route the data. Due to the unsecure and ad hoc 
nature of VANET, the network is prone to various security attacks that leads to devastating results. various security attacks have evolved that poses a threat to security of the network.

J.T. Isaac et al.[2] have discussed security threats and attacks that can be exploited in VANETs and has provided the corresponding security solutions to prevent those attacks.

Q.wu et al.[22] have proposed a new faithfull forwarding protocol in VANET which is supported by GeoDTN and navigation. They have used trust management model of Bayesian. these models consists of four steps1) routing starting 2) finding the routing 3) the faithful routing connection 4) the removal of routing. The proposed protocol not lowers time complexity and also improves routing security. The analysis showed that the method gives better performance and is able to remove ratio of intruder nodes, and receiver of correct packet ratio.

Seyed Mohammad Safi[21] have proposed an efficient method to prevent wormhole attack in vehicular ad hoc networks and tofind out malicious nodes. wormhole attack in vehicular network leads to significant loss and therefore AODV is considered as a routing protocol.. In this paper, they have used packet leashes and the method of authentication called HEAP is used. And they have also corrected packet leashes method. the proposed scheme can avoid wormhole attack in these types of networks .their suggested scheme has low overhead and increases security and performance to a significant level in the network.

\section{PROPOSED METHOD}

In recent years, there is rapid increase in the number of vehicles and it is becoming difficult to control the speed between vehicles and to maintain safe distance due to lack of attention by drivers and these challenges has led to the danger faced by drivers. These challenges require successful communication between vehicles with minimum delay. Various routing protocols have been designed and research is going on to improve the efficiency of the routing protocol. Transceivers and computerized control modules are needed in the vehicles to provide them with the ability to communicate as network node.

\section{A. FINE TUNED OLSR PROTOCOL}

The OLSR is a link state protocol for routing data packet developed for MANET and is very well adopted for VANET. Fine tuned OLSR protocol is the optimized standard OLSRprotocol by using meta heuristic algorithm as it is less affected by congestion problem.

The problem in OLSR with meta heuristic algorithm is with the parameters. Energy efficiency and Delay has reduced but somehow packet delivery ratio and throughput has not improved in significant ratio. Genetic algorithm can be used to get more efficient parameters.

\section{B. Genetic Algorithm}

Genetic algorithm is a metaheuristic approach that is based on the natural selection process. This approach is used tooptimize the various protocols by using techniques and methods inspired by natural evolution such as crosssover,mutation ,selection and inheritance.In this approach, an individual which consisits of population of candidate solution to an optimal problem paves a way towards better solution.each candidate solution possess certain set of properties that can be further altered and mutated.Fitness of every individual is calculated,which is the objective function of every problem.The fit indiviual is selected for further process

the genetic algorithm needs:

1.genetic representation of solution domain

\section{2.fitness function}

GA starts giving a population of solution once the genetic representation and fitness value is calculated and then enhance it with the use of repititive application of crosssover,mutation, selection and inheritance.

\section{STEPS OF ALGORITHM:}

\section{Initialization:}

$>$ Direction Vecor [DV]

$>$ Base station Set [BSS]

$>$ Tm; default; infinity; Coverage target$_{\text {; }}$; default; $\sum_{i}^{n}$ cvble $[i]$,field area

$>$ Sensor Data collection: After time instant $t_{\mathrm{m}}$ every node transmits the sensor vector to its router. Sensor_vector=( id, rx_power, energy, coverage $)$

\section{Recombination:}

fitness $(\mathrm{i})=($ coverage $[\mathrm{i}][\mathrm{r}]-$ coverage $[\mathrm{i}][\mathrm{r}-1])+$ (rx_power[i][r-1] - rx_power[i][t])- (energy[i][r-1] +energy[i][r-1] - energy m $_{\mathrm{m}}$ for $\mathrm{rx} \_$power[i][t] $\geq$ rx_powerWhere:

coverage is the coverage area,rx_power is the receiver power, and energy is the remaining energy.

\section{Mutation:}

$$
\begin{aligned}
& \text { if } \sum_{\boldsymbol{i}}^{\boldsymbol{n}} \boldsymbol{c} \boldsymbol{v} \boldsymbol{g}[\boldsymbol{i}][\boldsymbol{r}]-\sum_{\boldsymbol{i}}^{\boldsymbol{r}} \boldsymbol{c} \boldsymbol{v} \boldsymbol{g}[\boldsymbol{i}][\boldsymbol{r}-\mathbf{1}] \leq \\
& \operatorname{coverag}_{\boldsymbol{e}_{\text {delta }}} \text { mutate DV and BSS and decrement } \mathrm{t}_{\mathrm{m}} \text { by } \\
& \mathrm{t}_{\text {delta }}
\end{aligned}
$$

\section{Termination:}

If $\mathrm{t}_{\mathrm{m}}=0$ or $\sum_{i}^{n} \operatorname{cvg}[i][r] \geq$ coverage $e_{\text {target }}$ then terminate;else repeat from second step 
Table I. Comparison Of Protocols

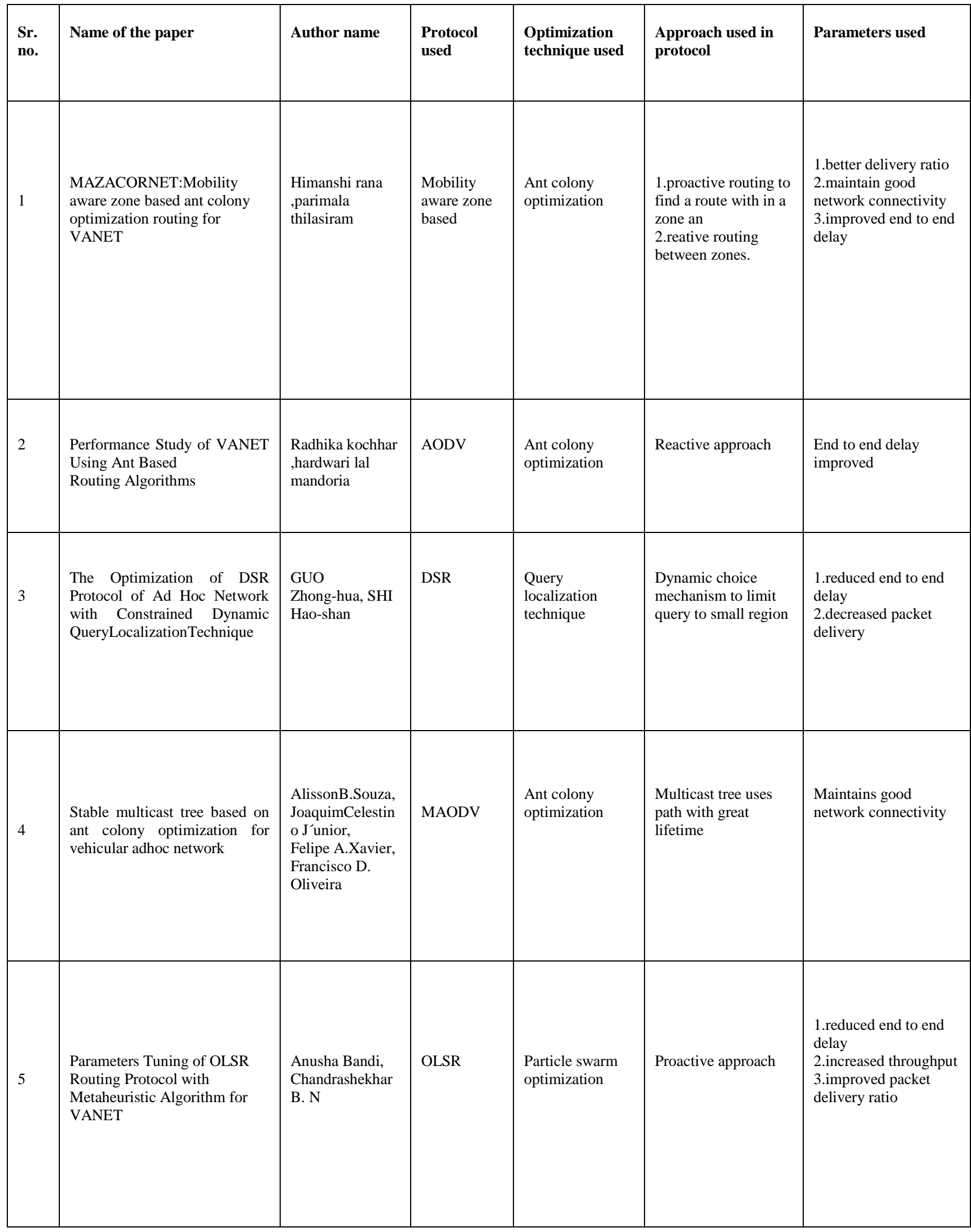




\section{V.RESULTS}

End to End Delay:It is the average time a packet takes to reach the destination.sometimes due to link failure,packet is not able to reach its destination and causes delay.

$$
\text { end to end delay }=\text { reception time }- \text { sent time }
$$

Table II. comparison between olsr and optimized olsr

\begin{tabular}{|l|l|l|}
\hline Time instant & $\begin{array}{l}\text { Energy spent of } \\
\text { olsr }\end{array}$ & $\begin{array}{l}\text { Energy Spent } \\
\text { of olsr+genetic }\end{array}$ \\
\hline $2 \mathrm{~s}$ & $1 \mathrm{~J}$ & $0 \mathrm{~J}$ \\
\hline $4 \mathrm{~s}$ & $4.8 \mathrm{~J}$ & $3.7 \mathrm{~J}$ \\
\hline $6 \mathrm{~s}$ & $3.24 \mathrm{~J}$ & $2.33 \mathrm{~J}$ \\
\hline $8 \mathrm{~s}$ & $1.8 \mathrm{~J}$ & $1.6 \mathrm{~J}$ \\
\hline $10 \mathrm{~s}$ & $1.7 \mathrm{~J}$ & $1.4 \mathrm{~J}$ \\
\hline
\end{tabular}

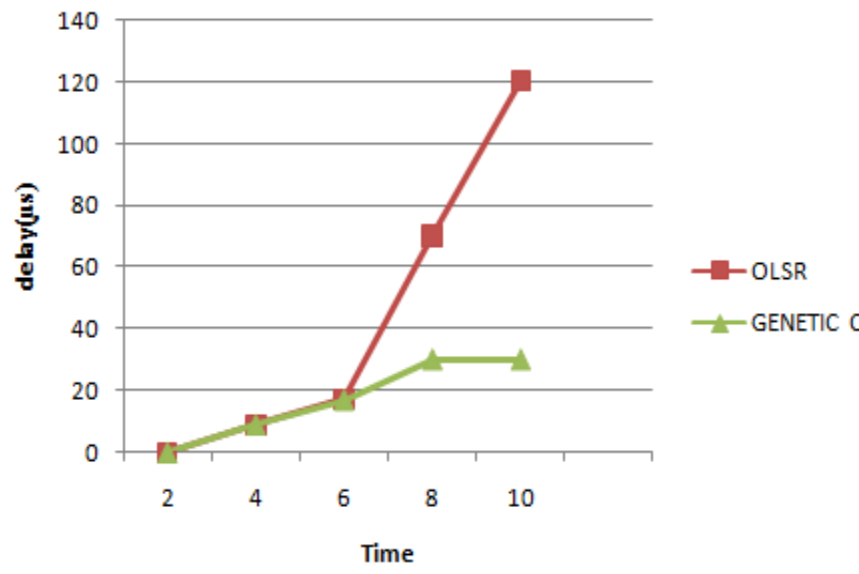

Figure 2.comparison of end to end delay

As observed from the Fig.1, it is clear that the delay that is experienced in the scenario of OLSR is 130 us wherein the delay in the case where OLSR+Genetic is used is 20 us that makes a huge difference. The reason behind this is the time saving in the process of sensing. With the use of genetic algorithm,coverage data is collected in the simulator from the record of displacement of nodes generated by the software.Using min_coverage and max_coverage values,an accurate loaction of the nodes can be found out which results in decrease in the end to end delay.Table 2 shows that at $0 \mathrm{~s}, 4 \mathrm{~s}, 6 \mathrm{~s}$ the end to end delay for OLSR and optimized OLSR is same which is 0us,9us and 17usrespectively because OLSR protocol takes 1.5 s to execute its functionality.

Energy Spent:Energy spent is consumption of energy or battery power.As we know in vehicle to vehicle. communication,battery power is used to conduct various operations so saving the battery power is one of the major issue.The formula of energy spent is:

$$
E\left(\frac{K W h}{d a y}\right)=P(W) * t(h / \text { day }) / 1000(W / k W)
$$

$1 \mathrm{kWh}=3600000$ Joule

where, $\mathrm{E}$ is energy in kilowatt-hours, $\mathrm{t}$ is hours per day, $\mathrm{P}$ is power in watts.

TableIII. comparison between olsr and optimized olsr

\begin{tabular}{|l|l|l|}
\hline Time instant & Delay of olsr & $\begin{array}{l}\text { Delay of } \\
\text { olsr+genetic }\end{array}$ \\
\hline $2 \mathrm{~s}$ & 0 us & 0us \\
\hline $4 \mathrm{~s}$ & $9 \mathrm{us}$ & 9 us \\
\hline $6 \mathrm{~s}$ & 17 us & 17 us \\
\hline $8 \mathrm{~s}$ & $70 \mathrm{us}$ & $30 \mathrm{us}$ \\
\hline $10 \mathrm{~s}$ & $120 \mathrm{us}$ & $30 \mathrm{us}$ \\
\hline
\end{tabular}

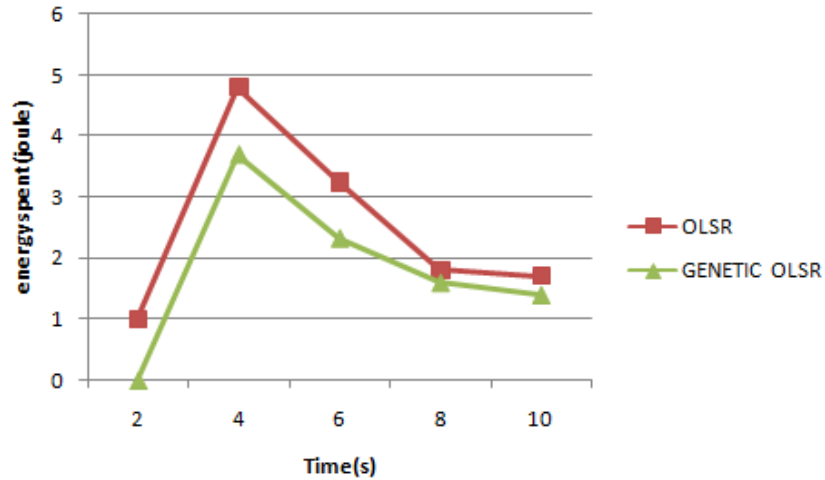

Figure 2. comparison of energy spent

As depicted in Fig.2 energy spent is quite clear that the energy spent in the case where OLSR and genetic are used is less than OLSR. The energy consumption in the proposed algorithm is $1.4 \mathrm{~J}$ wherein the energy consumed in OLSR is $1.7 \mathrm{~J}$. Energy consumotion is based on the nodes initial energy level.We have set initial energy as 90J.Once the simulation starts, after $1.5 \mathrm{~s}$ the nodes start transmitting packets and emits radiations.It emits more energy if nodes are moving hence gradually the energy decreases.In case of optimized OLSR algorithm nodes with excellent energy level gets selected and a path for the packet transmission is made among these nodes and has less chnaces of link breakage and hence energy will be conserved

\section{CONCLUSION}

The implementation of standard routing protocol with optimization technique such as particle swarm optimization, linear query search, genetic algorithm, ant colony optimization etc, has contributed in upgrading the efficiency of routing protocols. The parameters such as delivery ratio, end to end delay has shown significant improvement. Fine tuned OLSR protocol optimized by particle swarm optimization is less affected by problem of congestion. This protocol has efficiently reduced delay and energy efficiency but genetic algorithm can be used to get parameters that are more efficient.

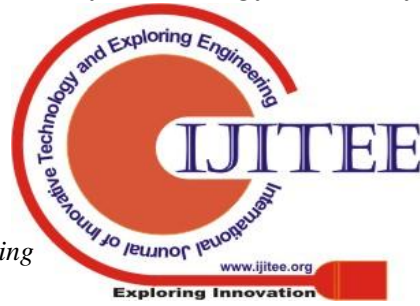




\section{Enhanced Routing Protocol for VANET}

\section{REFERENCES}

[1] S. Ganguly, S. Das, "A Novel Ant Colony Optimization Algorithm for the Vehicle Routing Problem." Swarm,Evolutionary and Memetic Computing, Springer, vol.8297, 2013, pp.401-412.

[2] Isaac, J. Téllez, S. Zeadally, and J. S. Camara, "Security attacks and solutions for vehicular ad hoc networks." Communications, IET 4.7, 2010, pp.894-903.

[3] Kochhar, Mandoria, "Performance study of VANET using ant based routing algorithms." Computing for Sustainable Global Development (INDIACom), 2nd International Conference, IEEE, New Delhi, 2015, pp.1803-1806.

[4] Mane and S. Kulkarni, "QoS realization for routing protocol on VANETs using combinatorial optimization."Computing, Communications and Networking Technologies (ICCCNT), Fourth International Conference, IEEE,Tiruchengode, 2013,pp.1-5.

[5] Y. Lin, Y. Chen and S. Lee, "Routing protocols in vehicular adhoc network:A survey and future perspectives." Journal of information science and engineering, vol.26, 2010, pp.913-932.

[6] Guo, Zhong-Hua, and Hao-Shan Shi. "The Optimization of DSR Protocol of Ad Hoc Network with Constrained Dynamic Query Localization Technique."2007 International Conference on Wireless Communications, Networking and Mobile Computing. IEEE, 2007,pp.1585-1588.

[7] Bandi, Anusha. "Parameters tuning of OLSR routing protocol with metaheuristic algorithm for VANET." Advance Computing Conference (IACC), 2015 IEEE International. IEEE 2015,pp.1207-1212.

[8] Rana, Hemant, P. Thulasiraman, and R. Thulasiram, "MAZACORNET: Mobility aware zone based ant colony optimization routing for VANET." Evolutionary Computation (CEC), IEEE, Cancun, 2013, pp.2948-2955.

[9] I. Sumra, Ahmed, H. Hasbullah, and J. AbManan., "Attacks on Security Goals (Confidentiality, Integrity, Availability) in VANET: A Survey." Vehicular Ad-hoc Networks for Smart Cities, Springer, Singapore, vol.306, 2015, pp.51-61.

[10] P. Stodola, J. Mazal, and M. Podhorec, "Improving the Ant Colony Optimization Algorithm for the Multi-Depot Vehicle Routing Problem and Its Application." Modelling and Simulation for Autonomous

Systems:FirstInternationalWorkshop,Springer,Rome,Vol.8906, 2014,pp.376-385.

[11] S. Shah, M. Shiraj, R. Noor, "Unicast routing protocols for urban vehicular networks: review, taxonomy, and open research issues." Journal of Zhejiang University, vol.15, 2014, pp. 489-513.

[12] Souza, A. B., Celestino, J., Xavier, F. A., Oliveira, F. D., Patel, A., \& Latifi, M."Stable multicast trees based on Ant Colony optimization for vehicular Ad Hoc networks." The International Conference on Information Networking 2013 (ICOIN). IEEE, 2013,pp.101-106.

[13] Mavrovouniotis, Michalis, and S. Yang, "Dynamic vehicle routing: A memetic ant colony optimization approach." Automated Scheduling and Planning, Springer, Berlin Heidelberg, 2013, pp. 283-301.

[14] S. Kumar, A. Kumar, "Position Based Routing Protocols in VANET: A Survey." Wireless Personal Communications , Springer US, vol.83, 2015, pp. 1-26.

[15] K. R. Jothi, A. Ebenezer, "Optimization and Quality-of-Service Protocols in VANETs: A Review." Artificial Intelligence and Evolutionary Algorithms in Engineering Systems, Springer India, vol.324, 2015, pp.275-284.

[16] D. Gaikwad, Sudhakar, and M. Zaveri, "Vanet routing protocols and mobility models: A survey." Trends in Network and Communications, Springer, Berlin Heidelberg, vol.197, 2011, pp.334-342.

[17] A. Baber, P. Wang, and C. Zou, "An economical, deployable and secure vehicular ad hoc network." In Military Communications Conference.IEEE,San Diego,CA, 2008,pp.1-7.

[18] F. Ali, F. Sheikh, A. Ansari, "Comparative Analysis of VANET Routing Protocols: On Road Side Unit Placement Strategies." Wireless Personal Communications, Springer, 2015, pp.1-14.

[19] M.Asgari, K.Jumari, M. Ismail, "Analysis of routing protocols in vehicular ad hoc network applications." Software Engineering and Computer Systems, Springer, Berlin Heidelberg, vol.181, 2011,pp.384-397.
[20] Safi, Seyed Mohammad, Ali Movaghar, and Misagh Mohammadizadeh. "A novel approach for avoiding wormhole attacks in VANET." 2009 First Asian Himalayas International Conference on Internet. IEEE, 2009,pp.1-6.

\section{AUTHORS PROFILE}

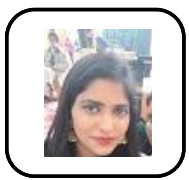

Ramneet kaur is a research scholar in computer science department in sri guru granth sahib world university.

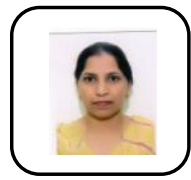

Dr.Navdeep kaur is a professor in computer science department in sri guru granth sahib world university.

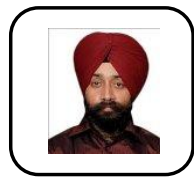

Dr.satwinder singh is associate professor in computer science department in central university of punjab,Bathinda. 